Article

\title{
Polish Yellow Sweet Clover (Melilotus officinalis L.) Honey, Chromatographic Fingerprints, and Chemical Markers
}

\author{
Izabela Jasicka-Misiak *, Ewa Makowicz and Natalia Stanek \\ Faculty of Chemistry, Opole University, Oleska 48, 45-052 Opole, Poland; makowicz.ewa@gmail.com (E.M.); \\ natalka0703@op.pl (N.S.) \\ * Correspondence: izajm@uni.opole.pl; Tel.: +48-077-452-7115
}

Academic Editor: Nancy D. Turner

Received: 15 November 2016; Accepted: 12 January 2017; Published: 15 January 2017

\begin{abstract}
A case study of Polish Melilotus officinalis honey was presented for the first time. Gas chromatography-mass spectrometry (GC-MS) (after steam distillation, Soxhlet extraction, ultrasonic solvent extraction, and solid phase extraction (SPE)) and targeted high performance liquid chromatography with a photodiode array detector (HPLC-PAD) were applied to determine the characteristic components of honey. While ubiquitous in most honeys, carbohydrates, terpene derivatives, and phenylacetic acid dominated in the Soxhlet extracts $(25.54 \%)$ and in the application of SPE (13.04\%). In addition, lumichrome (1.85\%) was found, and may be considered as a marker of this honey. Due to the presence of these compounds, Polish yellow sweet clover honey is similar to French lavender honeys. The major compounds determined in the methanolic extract were (+)-catechine (39.7\%) and gallic acid (up to 30\%), which can be regarded as specific chemical markers of the botanical origin of melilot honey. With respect to total phenolic and flavonoid contents, 1,1-diphenyl-2-picrylhydrazyl (DPPH) assays were determined spectrophotometrically. The honey exhibited a moderate antioxidant activity, typical for light honeys, which correlates well with its phenolic and flavonoid composition.
\end{abstract}

Keywords: yellow sweet clover; Melilotus officinalis; phenolic; volatile; honey markers

\section{Introduction}

Melilotus officinalis, also known as yellow melilot or yellow sweet clover, is a member of the Fabaceae family. It is a biennial aromatic herb, native to Europe and Asia, and has been considered a medicinal plant since ancient times. Hippocrates, the father of medicine, used sweet clover herb to treat skin ulcers, while Dioscorides described melilot as an emollient and anti-edematous drug. Bock recommended melilot for emollient ulcers, abscesses, and as an analgesic agent. The well-known French herbalist, Leclerc, described the sedative and antispasmodic actions of sweet clover [1]. In 19th century medicine, herb and flowering shoots were used to prepare mush compresses, whereas an herbal tea found application as a wash and rinse for swelling and swollen glands, abscesses, and swelling of the lymph nodes. Tea herbs, or the flowers themselves, were applied in cases of cold, mucosity, and respiratory and gastrointestinal catarrh [1].

In modern natural medicine, sweet clover is still used to support the treatment of many diseases. Extracts of this herb, containing coumarins (e.g., 7-hydroxycoumarin and 6,7-dihydroxycoumarin) are used in the treatment of phlebitis, in preventing thrombosis and vascular fragility [2], and in the treatment of varicose veins and hemorrhoids [3]. Sweet clover has sedative and antispasmodic effects towards the smooth muscles of the digestive tract, respiratory, and excretory systems, and also affects the effect of choleretics. M. officinalis extract is also used in the treatment of diabetic foot ulcers [2]. 
In addition to the reported medicinal properties, melilot is also used to add flavor and aroma to foods and beverages [4].

Yellow melilot smells like freshly-mown hay. All species of Melilotus, when flowering, have a peculiar sweet odor, which, through drying, becomes stronger and more agreeable. The characteristic sweet odor is derived from its coumarins (e.g., coumarin, 7-hydroxycoumarin and 6,7-dihydroxycoumarin). In Europe, dried Melilotus was traditionally placed in storage boxes and chests with clothing and linens in order to protect them from moths, in the same manner as dried lavender [5]. Medical sweet clover herb contains coumarin, which breaks down, when the plant is spoiled or damaged, into dicoumarin. This compound is used as a blood thinner and anticoagulant in rat and mouse poisons, and also for treating human ailments [6].

Recently, scientists and food producers have been more and more interested in plant-based foods [7-9]. These generally less-processed foods contain many valuable, biological active substances, with proven beneficial effects on consumers' health. Among others, bee-derived products, in particular honey, are very highly appreciated and recognized. Honey, beyond its flavor and nutritional value, is also known to have proven therapeutic properties, undoubtedly one of its most valuable qualities. This fact, not only provides many opportunities for the use of honey as an ingredient in many medicinal products, but also increases its value as an element of human diet. Therefore, integrated studies on the chemical composition, botanical origin, and biological activity of bee products are very important in the context of their use as an ingredient in modern food.

Polish Melilotus species (yellow and white sweet clover) blooms from June to late August, and are attractive and productive nectariferous plants. However, according to the best of our knowledge, the chemical composition and biological activity of yellow sweet clover honey have not yet been investigated. Therefore, the main objective of this study was to characterize Polish yellow sweet clover honey through investigation of the group of volatile and phenolic compounds that can be isolated from this product. The characterization was based, in each group, on the determination of its chromatographic fingerprint profile, and we attempted to identify the specific chemicals within the profile. Due to substantial differences in physicochemical properties between the two groups, two approaches were used in order to achieve this goal. For volatiles, isolation by either Soxhlet or ultrasonic extraction, as well as through steam distillation in the presence of an organic solvent, combined with a GC-MS analysis, was applied. To characterize a group of phenolic compounds, solid phase extraction was used, followed by analysis of the extract using HPLC-PAD (including the determination of total phenolic contents). Based on the obtained results, coumarin has been selected as a potential marker for this honey, and its content was quantified using GC-MS. Additionally, the determination of antioxidant properties of honey, utilizing DPPH assays, was performed.

\section{Results and Discussion}

\subsection{Volatile Compounds Identified by GC-MS}

GC-MS analysis of dichloromethane extracts, obtained after steam distillation, Soxhlet extraction, and ultrasonic solvent extraction (USE) of three Polish sweet yellow clover honeys led to the identification of 82 volatile compounds. These studies also showed that the applied methods for extraction appear to be suitable procedures for the isolation of volatiles from honey samples. Average peak areas of the identified compounds for honeys obtained from different years are shown in Table 1 . Chromatographic analysis of the extracts obtained using the solid phase extraction method, with the use of an SDBL cartridge, enabled the identification of 26 volatile compounds (Table 2). Volatile compounds present in Melilotus officinalis honey represent different groups of chemical entities; below we present the average content of the compounds present in the highest concentrations in all tested honey samples. When using the steam distillation technique, the highest contents for $\beta$-phellandrene $(2.17 \%)$, benzenacetaldehyde $(5.84 \%)$, phenylethyl alcohol $(2.03 \%), \beta$-menthane $(2.23 \%)$, thymol $(2.03 \%)$, and ethyl 2-(5-methyl-5-vinyltetrahydrofuran-2-yl)propan-2-ylcarbonate $(2.84 \%)$ were 
detected. When Soxhlet extraction was used, the highest contents for carophyllene (11.23\%), 13-epimanool (1.45\%), phenylacetic acid (5.35\%), and p-eugenol (6.54\%) were observed. While using USE, the predominant compounds were phenylacetic acid (25.54\%), $\alpha$-(phenylmethyl)benzenethanol $(6.44 \%)$, and $p$-acetoxyanisole (2.89\%). In the case of the SPE method, two compounds had the largest areas of peaks: Phenylacetic acid (13.04\%) and N-butylbenzensulfonamide (10.94\%). This method, however, provided the lowest number of compounds, and, thus, the results are incomparable with other methods. Coumarin is a volatile compound, which is not widespread among different varieties of honeys and, thus, is probably one of the most interesting compounds identified in sweet clover honey. This substance belongs to a group of naturally-occurring phenolic compounds that contain the characteristic benzo[ $\alpha]$ pyrone moiety and are produced via the shikimate pathway [10]. Coumarins are found in high levels in several plant species, such as the cinnamon tree, lavender, Prunus mahaleb L., and in sweet yellow clover [11-14]. It has been identified as a compound with very interesting pharmaceutical properties, such as the ability to increase blood flow in veins and decrease capillary permeability. Coumarin also poses antifungal and anti-cancer properties, but can be toxic when it is used at high doses for a long time [10]. The average content of coumarin in two-year-old Polish sweet yellow clover honey is $728 \mathrm{ng} / \mathrm{g}$ of honey, with the highest concentration being $902 \mathrm{ng} / \mathrm{g}$ of honey (see Table 3). Coumarin was not detected in fresh honey after harvesting (sample from 2016). The greatest amount of coumarin was obtained through steam distillation.

The results of other studies show that coumarin is also present in French lavender honeys, derived from Lavandula angustifolia Mill., Lavendula angustifolia $x$ latifolia, and Lavendula hybrid Reverchon II, where it mainly acts as a freshness indicator, and in honey from Prunus mahaleb L., where it serves as a nonspecific biomarker [12,13]. In lavender honeys, the average concentration of coumarin is lower in honeys analyzed after harvest (62 to $292 \mathrm{ng} / \mathrm{g}$ ) with a progressive increase during storage (from 512 to $1720 \mathrm{ng} / \mathrm{g}$ in one-year-old honey) [12,15]. The coumarin concentration in our two-year-old sweet yellow clover honey (2014) is lower than that in two-year-old lavender honey. These analytical data may suggest that the total content of coumarin in lavender honeys is higher than that in Polish sweet yellow clover honeys.

The second interesting compound, which was isolated using the SPE method, is lumichrome. This compound is a common breakdown product of riboflavin, which forms in the presence of light, in neutral or acidic solutions. In an alkaline solution, riboflavin is decomposed into lumiflavin. Other studies suggested that it could also be produced via an active mechanism, and cause different biochemical activities. Lumichrome plays an important regulatory role in plants by enhancing photosynthesis in soybean and corn, and is also probably responsible for seeding development in legumes and cereals. Thus far, there is no evidence that lumichrome has any important biological role in humans $[16,17]$. Lumichrome, for the first time, was identified in high concentrations in thistle (Galactites tomentosa Moench) honey, and, together with phenylacetic acid, was proposed as a marker for this monofloral honey [18]. Further studies have also shown the presence of lumichrome in other honeys: Dalmatian sage and cornflower (Centaurea cyanus L.) honeys, as well as a presence in other honeys in lower concentrations (Aarbutus unedo L., Asphodelus microcrapus, Citruss spp. Eucayptus spp., Hedysarum coronarium L., Salvia officinalis L., and Satureja spp.). It appears that, for honeys that contain lumichrome, the concentration of phenylacetic acid is higher than the normally observed in honey samples [17,19] with one exception-in Dalmatian sage honey, authors have demonstrated the presence of lumichrome, while phenylacetic acid was not found in the tested samples [17]. We have confirmed that lumichrome is present in Polish sweet yellow clover honey (Melilotus officinalis) and that it is accompanied by an enhanced concentration of phenylacetic acid. Obtained results suggest that the presence of coumarin in sweet yellow clover honey may be significant for botanical classification of this type of monofloral honey. Additionally, lumichrome and a high concentration of phenylacetic acid can be treated as potential markers. 
Table 1. Volatile compounds of Polish sweet yellow clover honey characterized by gas chromatography-mass spectrometry (GC-MS) in extracts obtained with different procedures.

\begin{tabular}{|c|c|c|c|c|c|c|c|c|c|c|}
\hline \multirow{4}{*}{ No. } & \multirow{4}{*}{ Compounds } & \multicolumn{9}{|c|}{ Peak Area (\%) } \\
\hline & & \multicolumn{3}{|c|}{ A } & \multicolumn{3}{|c|}{ B } & \multicolumn{3}{|c|}{ C } \\
\hline & & H14 & H15 & H16 & H14 & H15 & H16 & H14 & H15 & H16 \\
\hline & & \multicolumn{3}{|c|}{ Av. $(n=3)$} & \multicolumn{3}{|c|}{ Av. $(n=3)$} & \multicolumn{3}{|c|}{ Av. $(n=3)$} \\
\hline 1 & $(1 S, 15 S)$-Bicyclo[13.1.0]hexadecane & & & & $0.00 \pm 0.00$ & $0.69 \pm 0.09$ & $0.00 \pm 0.00$ & - & - & - \\
\hline 2 & $\beta$-Phellandrene & $1.96 \pm 0.21$ & $0.61 \pm 0.28$ & $1.36 \pm 0.45$ & - & - & - & - & - & - \\
\hline 3 & $\delta$-Selinene & $0.10 \pm 0.02$ & $0.12 \pm 0.05$ & $0.10 \pm 0.05$ & - & - & - & - & - & - \\
\hline 4 & 11-Tricosene & - & & - & $0.92 \pm 0.00$ & $0.92 \pm 0.20$ & $1.00 \pm 0.31$ & - & - & - \\
\hline 5 & 17-Pentatriacontene & 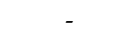 & & & & & & $0.70 \pm 0.23$ & $0.59 \pm 0.03$ & $0.56 \pm 0.12$ \\
\hline 6 & Docosene & $0.51 \pm 0.03$ & $0.56 \pm 0.04$ & $0.52 \pm 0.03$ & $0.96 \pm 0.05$ & $1.56 \pm 0.05$ & $1.60 \pm 0.12$ & $0.00 \pm 0.00$ & $1.48 \pm 0.63$ & $1.12 \pm 0.27$ \\
\hline 7 & 1-Heptacosanol & - & - & - & - & - & - & $1.46 \pm 0.65$ & $1.70 \pm 0.04$ & $1.51 \pm 0.63$ \\
\hline 8 & 1-Heptacosane & - & - & - & - & - & - & $1.06 \pm 0.26$ & $0.55 \pm 0.21$ & $0.59 \pm 0.06$ \\
\hline 9 & 1-Hexacosene & $0.71 \pm 0.09$ & $0.80 \pm 0.05$ & $0.69 \pm 0.10$ & - & - & - & - & - & - \\
\hline 10 & 13-Epimanool & - & - & - & $0.00 \pm 0.00$ & $2.15 \pm 0.98$ & $1.46 \pm 0.41$ & - & - & - \\
\hline 11 & 1-Nonadecene & $0.25 \pm 0.02$ & $0.25 \pm 0.01$ & $0.26 \pm 0.01$ & - & - & - & - & - & - \\
\hline 12 & 1-Oxa-spiro[4.5]deca-6,9-diene-2,8-dione & $0.32 \pm 0.10$ & $0.28 \pm 0.01$ & $0.28 \pm 0.03$ & - & - & - & - & - & - \\
\hline 13 & $\begin{array}{l}\text { 2,6,10,14-Tetramethyl } \\
\text {-7-(3-methylpent-4-enylidene) pentadecane }\end{array}$ & - & - & - & $0.96 \pm 0.00$ & $1.19 \pm 0.00$ & $0.00 \pm 0.00$ & - & - & - \\
\hline 14 & 2-Ethyl-5-n-propylphenol & - & - & - & $0.23 \pm 0.06$ & $0.27 \pm 0.16$ & $0.00 \pm 0.00$ & - & - & - \\
\hline 15 & 2-Ethylhexyl trans-4-methoxycinnamate & $0.51 \pm 0.02$ & $0.48 \pm 0.05$ & $0.00 \pm 0.00$ & - & - & - & - & - & - \\
\hline 16 & 2-Phenyl-3-(2-furyl)-propenal & $0.18 \pm 0.01$ & $0.19 \pm 0.01$ & $0.19 \pm 0.00$ & - & - & - & - & - & - \\
\hline 17 & 3,5-di-tert-Butyl-4-hydroxybenzaldehyde & $0.00 \pm 0.00$ & $0.09 \pm 0.01$ & $0.10 \pm 0.01$ & - & - & - & - & - & - \\
\hline 18 & 3-Furanmethanol/2-Furamethanol * & $0.41 \pm 0.02$ & $0.35 \pm 0.10$ & $0.39 \pm 0.20$ & - & - & - & - & - & - \\
\hline 19 & 5-Hydroxymethylfurfural & $5.94 \pm 1.20$ & $2.01 \pm 0.01$ & $1.12 \pm 0.09$ & $5.51 \pm 1.63$ & $4.32 \pm 1.97$ & $2.21 \pm 0.69$ & $15.21 \pm 4.21$ & $13.21 \pm 3.28$ & $10.26 \pm 1.96$ \\
\hline 20 & (E)-9-Octadecenoic acid ** & $6.98 \pm 1.02$ & $7.00 \pm 1.25$ & $5.25 \pm 0.96$ & $0.96 \pm 0.36$ & $1.80 \pm 0.64$ & $1.46 \pm 0.26$ & $1.64 \pm 0.23$ & $3.60 \pm 0.76$ & $2.01 \pm 0.36$ \\
\hline 21 & (Z)-9-Tricosene, & $1.26 \pm 0.69$ & $1.39 \pm 0.78$ & $1.33 \pm 0.20$ & - & - & - & $0.90 \pm 0.23$ & $0.89 \pm 0.41$ & $0.65 \pm 0.09$ \\
\hline 22 & Acetophenone & $0.00 \pm 0.00$ & $0.13 \pm 0.08$ & $0.19 \pm 0.10$ & - & - & - & - & - & - \\
\hline 23 & Behenic alcohol & & & & $0.78 \pm 0.22$ & $1.03 \pm 0.12$ & $0.00 \pm 0.00$ & - & - & - \\
\hline 24 & Benzaldehyde & $0.19 \pm 0.06$ & $0.25 \pm 0.08$ & $0.21 \pm 0.42$ & 2 & - & - & - & - & - \\
\hline 25 & 4-Octyl-N-(4-octylphenyl)benzenamine & & & & $1.23 \pm 0.13$ & $2.03 \pm 0.29$ & $1.63 \pm 0.09$ & - & - & - \\
\hline 26 & Benzeneacetaldehyde & $5.69 \pm 0.17$ & $6.02 \pm 0.10$ & $5.50 \pm 0.17$ & - & - & - & - & - & - \\
\hline 27 & Phenylacetic acid & - & - & - & $4.13 \pm 0.06$ & $6.14 \pm 0.65$ & $5.23 \pm 0.91$ & $28.12 \pm 3.74$ & $26.21 \pm 2.78$ & $24.26 \pm 3.65$ \\
\hline 28 & $\alpha$-(Phenylmethyl)benzeneethanol & - & - & - & - & - & - & $5.59 \pm 1.61$ & $8.80 \pm 0.09$ & $6.01 \pm 1.23$ \\
\hline 29 & Benzoic acid & - & - & - & - & - & - & $1.15 \pm 0.07$ & $0.85 \pm 0.37$ & $1.06 \pm 0.33$ \\
\hline 30 & 4-Hydroxy-3,5-dimethoxybenzoic acid & - & - & - & - & - & - & $1.78 \pm 0.23$ & $1.85 \pm 0.64$ & $1.79 \pm 0.36$ \\
\hline 31 & Benzothiazole & $1.21 \pm 0.19$ & $1.32 \pm 0.09$ & $1.30 \pm 0.09$ & - & - & - & - & - & - \\
\hline 32 & Benzyl alcohol & $0.25 \pm 0.02$ & $0.42 \pm 0.10$ & $0.40 \pm 0.03$ & - & - & - & - & - & - \\
\hline 33 & 2-Methylene4,8,8-trimethyl-4-vinyl-bicyclo[5.2.0]nonane & - & - & - & $0.96 \pm 0.63$ & $2.93 \pm 0.23$ & $2.21 \pm 0.09$ & - & - & - \\
\hline 34 & Bis(2-ethylhexyl) maleate & - & - & - & $1.01 \pm 0.56$ & $1.53 \pm 0.23$ & $1.26 \pm 0.09$ & - & - & - \\
\hline 35 & Caryophyllene & - & - & - & $13.21 \pm 3.31$ & $12.26 \pm 0.91$ & $13.60 \pm 3.69$ & - & - & - \\
\hline 36 & cis-13-Octadecenoic acid/trans-13-Octadecenoic acid ** & - & - & - & $1.71 \pm 0.63$ & $2.00 \pm 0.06$ & $1.96 \pm 0.06$ & - & - & - \\
\hline 37 & Coumarin & $1.41 \pm 0.15$ & $0.98 \pm 0.09$ & $0.00 \pm 0.00$ & $0.22 \pm 0.12$ & $0.19 \pm 0.09$ & $0.00 \pm 0.00$ & - & - & - \\
\hline 38 & Cyclohept-4-enone & $0.21 \pm 0.04$ & $0.19 \pm 0.10$ & $0.15 \pm 0.10$ & - & - & - & - & - & - \\
\hline
\end{tabular}


Table 1. Cont.

\begin{tabular}{|c|c|c|c|c|c|c|c|c|c|c|}
\hline \multirow{4}{*}{ No. } & \multirow{4}{*}{ Compounds } & \multicolumn{9}{|c|}{ Peak Area (\%) } \\
\hline & & \multicolumn{3}{|c|}{ A } & \multicolumn{3}{|c|}{ B } & \multicolumn{3}{|c|}{ C } \\
\hline & & H14 & H15 & H16 & H14 & H15 & H16 & H14 & H15 & H16 \\
\hline & & \multicolumn{3}{|c|}{ Av. $(n=3)$} & \multicolumn{3}{|c|}{ Av. $(n=3)$} & \multicolumn{3}{|c|}{ Av. $(n=3)$} \\
\hline 39 & $\beta$-Menthane & $1.85 \pm 0.35$ & $2.61 \pm 0.15$ & $2.52 \pm 0.31$ & - & & & - & - & - \\
\hline 40 & Cyclopentadecane & - & - & $-2=$ & $0.65 \pm 0.26$ & $0.95 \pm 0.06$ & $0.71 \pm 0.20$ & - & - & - \\
\hline 41 & Docosane & - & - & - & $1.36 \pm 0.23$ & $1.60 \pm 0.16$ & $1.09 \pm 0.21$ & - & - & - \\
\hline 42 & Dodecanoic acid & $0.38 \pm 0.21$ & $0.28 \pm 0.08$ & $0.00 \pm 0.00$ & - & - & - & - & - & - \\
\hline 43 & $E$-15-Heptadecenal & $0.41 \pm 0.20$ & $0.38 \pm 0.09$ & $0.53 \pm 0.20$ & - & - & - & - & - & - \\
\hline 45 & Eicosane & $2.65 \pm 0.43$ & $3.45 \pm 0.45$ & $4.41 \pm 1.01$ & $0.87 \pm 0.61$ & $1.31 \pm 0.26$ & $1.16 \pm 0.31$ & $1.86 \pm 0.71$ & $1.90 \pm 0.06$ & $1.74 \pm 0.21$ \\
\hline 46 & Eicosane/Hexadecan/Octadecane * & $1.62 \pm 0.98$ & $1.76 \pm 0.02$ & $1.82 \pm 0.63$ & $0.59 \pm 0.27$ & $0.91 \pm 0.24$ & $0.70 \pm 0.36$ & $3.28 \pm 1.21$ & $3.56 \pm 1.96$ & $0.00 \pm 0.00$ \\
\hline 47 & $\begin{array}{l}\text { Ethyl 2-(5-methyl-5-vinyltetrahydrofuran-2-yl) } \\
\text { propan-2-yl carbonate }\end{array}$ & $2.61 \pm 0.13$ & $2.91 \pm 0.65$ & $2.79 \pm 0.09$ & $1.03 \pm 0.52$ & $1.44 \pm 0.09$ & $1.26 \pm 0.47$ & - & - & - \\
\hline 48 & Ethyl oleate & $4.91 \pm 0.96$ & $6.21 \pm 1.21$ & $5.81 \pm 1.12$ & - & - & - & $2.00 \pm 0.57$ & $1.68 \pm 0.03$ & $1.80 \pm 0.07$ \\
\hline 49 & Furfural & $2.09 \pm 0.21$ & $1.95 \pm 0.09$ & $1.21 \pm 0.51$ & - & - & - & - & - & - \\
\hline 50 & Heneicosane & $2.09 \pm 0.20$ & $1.61 \pm 0.51$ & $1.82 \pm 0.91$ & - & - & - & $0.98 \pm 0.14$ & $1.06 \pm 0.08$ & $0.50 \pm 0.12$ \\
\hline 51 & Hentriacontane & - & - & - & - & - & - & $0.80 \pm 0.36$ & $0.77 \pm 0.21$ & $0.80 \pm 0.46$ \\
\hline 52 & Heptacosyl acetate & - & - & - & - & - & - & $1.69 \pm 0.41$ & $0.00 \pm 0.00$ & $1.56 \pm 0.98$ \\
\hline 53 & Heptadecane & $3.70 \pm 0.61$ & $4.42 \pm 0.91$ & $4.02 \pm 0.98$ & - & - & - & - & - & - \\
\hline 54 & Heptadecane/Hexacosane & - & - & 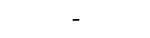 & - & - & - & $2.21 \pm 0.97$ & $1.98 \pm 0.06$ & $2.04 \pm 0.14$ \\
\hline 55 & Methyl hexadecanoate & $0.13 \pm 0.09$ & $0.00 \pm 0.00$ & $0.00 \pm 0.00$ & - & - & & & & \\
\hline 56 & Bis(2-ethylhexyl) hexanedioate & - & - & - & $3.36 \pm 0.61$ & $6.05 \pm 0.78$ & $4.90 \pm 1.76$ & $1.41 \pm 0.36$ & $2.06 \pm 0.26$ & $2.01 \pm 0.96$ \\
\hline 57 & Humulene & - & - & - & $0.63 \pm 0.23$ & $0.95 \pm 0.07$ & $0.77 \pm 0.31$ & & & - \\
\hline 58 & Methyl dehydroabietate & $0.61 \pm 0.36$ & $0.00 \pm 0.00$ & $0.21 \pm 0.00$ & $0.96 \pm 0.09$ & $1.16 \pm 0.45$ & $1.06 \pm 0.03$ & $0.00 \pm 0.00$ & $0.35 \pm 0.21$ & $0.00 \pm 0.00$ \\
\hline 59 & n-Hexadecanoic acid & - & - & - & $2.79 \pm 0.81$ & $4.00 \pm 1.15$ & $3.01 \pm 0.05$ & $2.90 \pm 0.21$ & $2.86 \pm 0.06$ & $1.16 \pm 0.31$ \\
\hline 60 & Nonacosane & - & - & - & $2.39 \pm 0.27$ & $2.78 \pm 0.97$ & $2.61 \pm 0.61$ & $0.91 \pm 0.06$ & $1.00 \pm 0.09$ & $0.90 \pm 0.36$ \\
\hline 61 & Nonadecane & $0.20 \pm 0.05$ & $0.15 \pm 0.06$ & $0.35 \pm 0.12$ & $0.37 \pm 0.21$ & $0.49 \pm 0.08$ & $0.41 \pm 0.23$ & - & - & - \\
\hline 62 & 9-Methylnonadecane, & - & - & - & $1.00 \pm 0.05$ & $1.70 \pm 0.07$ & $1.40 \pm 0.61$ & - & - & - \\
\hline 63 & Nonadecyl trifluoroacetate & - & - & - & $0.41 \pm 0.26$ & $0.90 \pm 0.14$ & $0.85 \pm 0.16$ & $1.44 \pm 0.02$ & $0.71 \pm 0.21$ & $1.01 \pm 0.36$ \\
\hline 64 & Octacosane & $0.91 \pm 0.32$ & $1.26 \pm 0.09$ & $1.09 \pm 0.26$ & $0.65 \pm 0.25$ & $0.80 \pm 0.04$ & $0.23 \pm 0.10$ & & & \\
\hline 65 & Octadecane & $0.98 \pm 0.21$ & $1.79 \pm 0.09$ & $1.38 \pm 0.31$ & $1.29 \pm 0.36$ & $1.46 \pm 0.31$ & $0.00 \pm 0.00$ & $1.35 \pm 0.41$ & $2.36 \pm 0.07$ & $1.70 \pm 0.36$ \\
\hline 66 & Octadecanoic acid & - & - & - & $2.13 \pm 1.71$ & $2.50 \pm 0.23$ & $2.41 \pm 0.96$ & - & - & -0 \\
\hline 67 & Oleic acid & - & - & - & $0.00 \pm 0.00$ & $1.53 \pm 0.69$ & $1.49 \pm 0.27$ & - & - & - \\
\hline 68 & $p$-Acetoxyanisole & - & - & & & & & $0.00 \pm 0.00$ & $3.21 \pm 1.25$ & $2.01 \pm 0.09$ \\
\hline 69 & $p$-Cymen-8-ol & $0.00 \pm 0.00$ & $0.21 \pm 0.09$ & $0.35 \pm 0.03$ & - & - & - & & - & - \\
\hline 70 & $p$-Eugenol & - & - & - & $5.65 \pm 1.21$ & $7.20 \pm 2.26$ & $6.20 \pm 0.91$ & $2.26 \pm 0.78$ & $1.96 \pm 0.21$ & $2.06 \pm 0.67$ \\
\hline 71 & 2-Methoxy-4-(1-propenyl)phenol & - & - & - & $0.56 \pm 0.07$ & $0.63 \pm 0.12$ & $0.59 \pm 0.12$ & $2.81 \pm 1.21$ & $3.60 \pm 0.94$ & $3.04 \pm 0.41$ \\
\hline 72 & Phenylethyl alcohol & $1.91 \pm 0.21$ & $0.26 \pm 0.05$ & $2.01 \pm 0.12$ & & & & - & - & - \\
\hline 73 & Supraene/Squalene* & $0.81 \pm 0.36$ & $1.28 \pm 0.14$ & $1.13 \pm 0.09$ & $2.71 \pm 1.21$ & $2.80 \pm 0.08$ & $2.67 \pm 0.08$ & - & - & - \\
\hline 74 & Tetracosane & - & - & - & & & & - & - & - \\
\hline 75 & Tetradecanoic acid & $0.21 \pm 0.10$ & $0.98 \pm 0.40$ & $0.41 \pm 0.21$ & $0.66 \pm 0.12$ & $1.20 \pm 0.36$ & $0.96 \pm 0.23$ & - & - & - \\
\hline 76 & Tetradecyl trifluoroacetate & - & - & - & $0.49 \pm 0.09$ & $0.50 \pm 0.31$ & $0.49 \pm 0.09$ & - & - & - \\
\hline 77 & Thymol & $2.76 \pm 0.09$ & $2.61 \pm 0.12$ & $2.85 \pm 0.01$ & - & - & - & - & - & - \\
\hline 78 & Tricosane & - & - & - & $0.98 \pm 0.23$ & $1.65 \pm 0.07$ & $1.12 \pm 0.36$ & - & - & - \\
\hline 79 & 2,3,4,5-Tetramethyl-tricyclo[3.2.1.02,7]oct-3-ene & $0.12 \pm 0.06$ & $0.25 \pm 0.06$ & $0.18 \pm 0.09$ & - & - & & - & - & - \\
\hline 80 & Z-10-Tetradecen-1-ol acetate & - & - & - & $0.33 \pm 0.09$ & $0.48 \pm 0.06$ & $0.40 \pm 0.20$ & - & - & - \\
\hline 81 & Z-12-Pentacosene & $0.20 \pm 0.09$ & $0.79 \pm 0.20$ & $0.71 \pm 0.36$ & $1.12 \pm 0.23$ & $0.69 \pm 0.02$ & $0.60 \pm 0.01$ & - & - & - \\
\hline 82 & $\alpha$-Terpinolene & $0.00 \pm 0.00$ & $0.42 \pm 0.00$ & $0.00 \pm 0.00$ & - & - & - & - & - & - \\
\hline
\end{tabular}

A—steam distillation, B-Soxhlet extraction, C-USE; * Tentatively identified; ** Correct isomer not identified; - Not identified; Av, = average; H14 = honey sample from 2014

H15 = honey sample from 2015, H16 = honey sample from 2016. 
Table 2. Volatile compounds identified by GC-MS in extracts obtained using solid phase extraction (SPE).

\begin{tabular}{|c|c|c|c|c|}
\hline \multirow{3}{*}{ No. } & \multirow{3}{*}{ Compounds } & \multicolumn{3}{|c|}{ Peak Area (\%) } \\
\hline & & H14 & H15 & H16 \\
\hline & & Av. $(n=3)$ & Av. $(n=3)$ & Av. $(n=3)$ \\
\hline 1 & trans-Linallol oxide/cis-Linallol oxide ** & $0.33 \pm 0.02$ & $0.00 \pm 0.00$ & $0.28 \pm 0.04$ \\
\hline 2 & Phynylethylalcohol & $0.10 \pm 0.03$ & $0.16 \pm 0.06$ & $0.00 \pm 0.00$ \\
\hline 3 & Hotrienol & $0.20 \pm 0.09$ & $0.08 \pm 0.02$ & $0.00 \pm 0.00$ \\
\hline 4 & $p$-cymen-8-ol & $0.16 \pm 0.09$ & $0.48 \pm 0.2$ & $0.09 \pm 0.01$ \\
\hline 5 & Benzoic acid & $0.00 \pm 0.00$ & $0.82 \pm 0.09$ & $0.12 \pm 0.09$ \\
\hline 6 & Phenylacetic acid & $6.21 \pm 1.21$ & $19.21 \pm 0.21$ & $17.65 \pm 2.24$ \\
\hline 7 & $\alpha$-Methylbenzeneethanol & $0.19 \pm 0.09$ & $3.2 \pm 0.91$ & $4.75 \pm 1.21$ \\
\hline 8 & Ethyl 4-ethoxybenzoate & $0.35 \pm 0.09$ & $0.51 \pm 0.2$ & $0.63 \pm 0.31$ \\
\hline 9 & $N$-Butylbenzenesulfonamide & $9.64 \pm 0.06$ & $12.06 \pm 1.25$ & $12.65 \pm 4.52$ \\
\hline 10 & 9-Hexadecanoic acid & $0.96 \pm 0.23$ & $0.00 \pm 0.00$ & $1.17 \pm 0.42$ \\
\hline 11 & Estra-1,3,5(10)-trien-17-ol & $0.96 \pm 0.25$ & $3.12 \pm 1.09$ & $2.59 \pm 0.05$ \\
\hline 12 & Oleic acid & $1.83 \pm 0.06$ & $1.90 \pm 0.36$ & $2.01 \pm 0.08$ \\
\hline 13 & $\begin{array}{l}\text { Octadecanoic acid/cis-13-Octadecenoic acid/ } \\
\text { cis-Vaccenic acid } * / * *\end{array}$ & $1.45 \pm 0.91$ & $1.85 \pm 0.03$ & $1.90 \pm 0.35$ \\
\hline 14 & 1-Docosene & $2.08 \pm 0.31$ & $2.40 \pm 0.39$ & $1.99 \pm 0.03$ \\
\hline 15 & (Z)-9-Tricosene & $0.75 \pm 0.03$ & $0.89 \pm 0.21$ & $0.72 \pm 0.01$ \\
\hline 16 & 1-Heptatriacotanol & $0.00 \pm 0.00$ & $0.84 \pm 0.02$ & $0.00 \pm 0.00$ \\
\hline 17 & $\begin{array}{l}\text { 2-Ehylhexyl } \\
\text { 3-(4-methoxyphenyl)-2-propenoate }\end{array}$ & $0.71 \pm 0.09$ & $0.71 \pm 0.21$ & $0.69 \pm 0.06$ \\
\hline 18 & Methyl dehydroabietate & $0.84 \pm 0.09$ & $0.51 \pm 0.10$ & $0.79 \pm 0.05$ \\
\hline 19 & (Z)-9-Octadecenamide & $1.81 \pm 0.36$ & $3.25 \pm 0.51$ & $2.25 \pm 0.95$ \\
\hline 20 & $\begin{array}{l}\text { 1,7,11-Trimethyl-4-(1-methylethyl) } \\
\text {-cyclotetradecane }\end{array}$ & $0.00 \pm 0.00$ & $0.81 \pm 0.32$ & $0.00 \pm 0.00$ \\
\hline 21 & Pentacosane & $0.90 \pm 0.21$ & $1.10 \pm 0.01$ & $1.01 \pm 0.32$ \\
\hline 22 & Nonadecane & $0.70 \pm 0.01$ & $1.29 \pm 0.21$ & $0.75 \pm 0.01$ \\
\hline 23 & Lumichrome & $1.82 \pm 0.30$ & $1.89 \pm 0.09$ & $1.86 \pm 0.15$ \\
\hline 24 & Squalene & $1.43 \pm 0.09$ & $1.62 \pm 0.21$ & $1.59 \pm 0.21$ \\
\hline 25 & Nonadecyl trifluoroacetate & $0.39 \pm 0.21$ & $0.51 \pm 0.09$ & $0.49 \pm 0.19$ \\
\hline 26 & Eicosane & $0.48 \pm 0.23$ & $2.18 \pm 0.23$ & $1.33 \pm 0.91$ \\
\hline
\end{tabular}

* Tentatively identified; ${ }^{* *}$ Correct isomer not identified; Av. = average; H14 = honey sample from 2014, H15 = honey sample from 2015, H16 = honey sample from 2016.

Table 3. Coumarin content in Polish sweet yellow clover honey.

\begin{tabular}{|c|c|c|c|c|c|c|c|c|c|c|}
\hline \multirow{4}{*}{ Method } & \multirow{4}{*}{$\mathbf{R}^{2}$} & \multicolumn{9}{|c|}{ Content of Coumarin } \\
\hline & & \multicolumn{9}{|c|}{ ng/g of Honey } \\
\hline & & \multicolumn{3}{|c|}{ H14 } & \multicolumn{3}{|c|}{ H15 } & \multicolumn{3}{|c|}{ H16 } \\
\hline & & Min & Max & Avg & Min & $\operatorname{Max}$ & Avg & Min & Max & Avg \\
\hline Steam distillation & 0.994 & 491.79 & 902.79 & 728.66 & 339.82 & 629.61 & 501.23 & \multicolumn{3}{|c|}{ Not detected } \\
\hline
\end{tabular}

Min, Max, Avg = minimal, maximal, average concentration of coumarin in honey samples; H14 = honey sample from 2014, H15 = honey sample from 2015, H16 = honey sample from 2016.

\subsection{Phenolic Compounds}

One of the aims of this study was to show and compare antioxidant activity, and the phenolic and flavonoids contents of yellow sweet clover honey. To the best our knowledge, chemical composition and antioxidant activity of this branch of honey has not yet been investigated.

\subsubsection{Total Phenolic and Flavonoids Content (TPC and TFC)}

According to color, honeys are generally divided into dark and light types. The first category includes honeys such as heather, oak, and chestnut, and, indeed, these honeys have the highest content of phenolic compounds compared to the second group, light-colored honeys, such as acacia, rape, goldenrod, and rhododendron, and they have significantly lower contents of phenolic compounds $[20,21]$.

It is well known that the TPC level of honeys exist in a wide range, from $0.5 \mathrm{mg} / 100 \mathrm{~g}$ to $130 \mathrm{mg} / 100 \mathrm{~g}$ of honey. Flavonoids constitute approximately $5 \%$ of these phenolic compounds. 
The amount of total phenolic content in Polish yellow sweet clover honey was measured using the Folin-Ciocalteu reagent method (Table 4) and was expressed as gallic acid equivalent (GAE). Phenolic content varied from $54.06 \mathrm{mg}$ to $55.51 \mathrm{mg}$ GAE/100 $\mathrm{g}$ of honey. The results obtained in the present study also showed that the values are within the ranges obtained for lavender honey $(53.39 \mathrm{mg}$ GAE/100 g), lime honey (41.20 mg GAE/100 g) [21], sunflower honey (54.63 mg GAE/100 g), sage honey (55.40 mg GAE/100 g), and goldenrod honey (49.24 mg GAE/100 g) [22].

The TFC of yellow sweet clover honey was expressed as quercetin equivalents and varied from $2.29 \mathrm{mg}$ to $2.39 \mathrm{mg} \mathrm{QE} / 100 \mathrm{~g}$ of honey. As above, the TFC is similar to that of lavender honey (2.20 $\mathrm{mg}$ QE/100 g) [21].

\subsubsection{HPLC-PAD Analysis of Honey Samples}

The HPLC analysis of sweet clover honey samples indicated the presence of 15 phenolic compounds; nine phenolic acids: gallic, 4-hydroxybenzoic, caffeic, 3-hydroxybenzoic, $p$-coumaric, ferulic, rosmarinic, ellagic, and cinnamic; and six flavonoids: (+)-catechin, myricetin, quercetin, genistein, pinocembrin, and morin. A representative HPLC chromatogram recorded at $280 \mathrm{~nm}$ is shown in Figure 1.

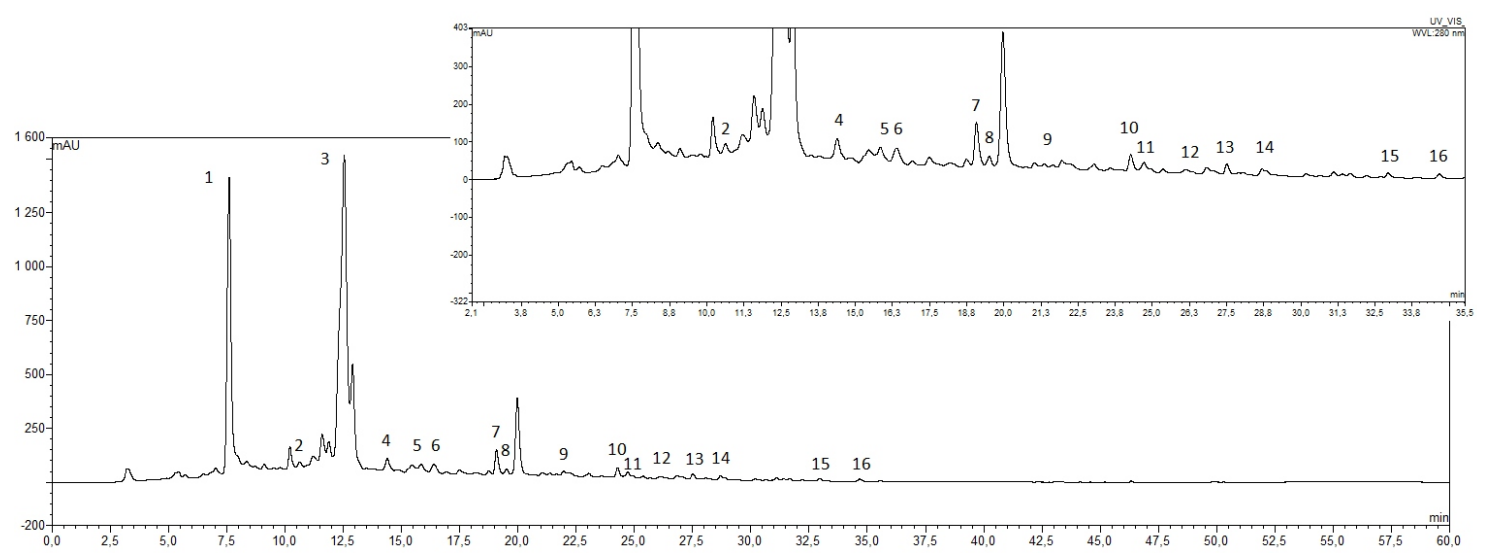

Figure 1. High performance liquid chromatography (HPLC) chromatograms of phenolic compounds in methanolic extracts of Polish yellow sweet clover honeys. Identified compounds are: (1) gallic acid; (2) 3,4-dihydroxybenzoic acid; (3) (+)-catechin; (4) 4-hydroxybenzoic acid; (5) caffeic acid; (6) 3-hydroxybenzoic acid; (7) $p$-coumaric acid; (8) ferulic acid; (9) rosmarinic acid; (10) ellagic acid; (11) myricetin; (12) cinnamic acid; (13) quercetin; (14) genstein; (15) pinocembrin; and (16) morin hydrate.

The calculated levels of individual identified phenolic compounds are shown in Table 4. Targeted HPLC-PAD analysis indicated the presence of significant amounts of gallic acid (23.23 $\pm 4.52 \mathrm{mg} /$ $100 \mathrm{~g}$ honey) and (+)-catechin (26.79 $\pm 2.99 \mathrm{mg} / 100 \mathrm{~g}$ honey).

Phenolic acids represent almost $30.0 \%$ of the total identified phenolic compounds present in each of the analyzed samples. The remaining determined compounds were flavonoids at a level of only about $5 \%$ of identified phenolic compounds, which is in accordance with the literature data.

All of the identified compounds have been previously found in various monofloral honeys. Gallic acid (1.35 mg to $11.27 \mathrm{mg} \mathrm{GAE} / 100 \mathrm{~g}$ ) and caffeic acid ( $0.41 \mathrm{mg}$ to $1.60 \mathrm{mg}$ GAE/100 g) were considered as markers for the floral origin of lavender honey [23]. Much smaller quantities of catechin (3.67 mg GAE/100 g) and p-coumaric acid (0.103 mg GAE/100 g) were identified in manuka honey [24]. Gallic acid (0.86 mg GAE/100 g), p-coumaric acid (0.30 mg GAE/100 g), ellagic acid (0.56 mg GAE/100 g), and quercetin (15.89 mg GAE/100 g) were found in in gelam honey [25]. It is worth mentioning that the levels of gallic acid or catechin are lower than in yellow sweet clover honey. Additionally, gallic acid and catechin contents are significant if compared to the contents of the other phenolics. Thus, they might be considered as characteristic compounds of the studied honey. 
Table 4. The level of individual identified phenolic compounds (mg/100 g of product) and content of total phenolics (GAE-gallic acid equivalent) and flavonoids. (QE-quercetin equivalent) compounds in Polish yellow sweet clover honeys (methanolic extract).

\begin{tabular}{|c|c|c|c|c|c|c|c|c|c|c|c|c|c|c|c|c|c|c|}
\hline $\begin{array}{l}\text { Phenolic } \\
\text { Compounds }\end{array}$ & GA & C & 4-HBA & CA & 3-HBA & $p$-CA & FA & RA & EA & MC & CiA & QC & G & $\mathbf{P}$ & MH & \multirow{2}{*}{$\begin{array}{l}\text { Total Phenolic } \\
\text { Content (mg } \\
\text { GAE/100 g) } n=2\end{array}$} & \multirow{2}{*}{$\begin{array}{l}\text { Total Flavonoids } \\
\text { Content (mg } \\
\text { QE/100 g) } n=2\end{array}$} & \multirow{2}{*}{$\begin{array}{c}\text { Radical Scavenging } \\
\text { Activity } \\
\text { DPPH (\%) } n=2\end{array}$} \\
\hline \multicolumn{16}{|c|}{$\begin{array}{l}\text { Levels of Individual Identified Compounds of Tested Honeys } \\
\text { (mg/100 g of Product) (RSD } \leq 5 \%, n=3 \text { ) }\end{array}$} & & & \\
\hline mean & 23.23 & 26.79 & 0.68 & 0.41 & 0.32 & 1.46 & 0.25 & 0.19 & 0.48 & 0.36 & 0.16 & 0.28 & 0.26 & 0.11 & 0.13 & 67.55 & 2.29 & 55.96 \\
\hline$\pm \mathrm{SD}$ & 4.52 & 2.99 & 0.43 & 0.11 & 0.02 & 0.12 & 0.03 & 0.04 & 0.03 & 0.05 & 0.01 & 0.03 & 0.07 & 0.01 & 0.03 & 7.51 & 0.11 & 2.35 \\
\hline
\end{tabular}

EA-ellagic acid, MC-myricetin, CiA—cinnamic acid, QC-quercetin, G-genistein, $\mathrm{P}$ - pinocembrin, MH—morin hydrate (mean values from three repetitions). 


\subsubsection{Antiradical Activity (DPPH Test)}

Antioxidant activity was measured by using a DPPH-based procedure. Literature data indicate that dark honeys are highly active as radical scavengers, more active than light honeys [26,27]. Additionally, antiradical activity depends on the location of floral sources of honey (botanical origin) [28]. The result of the DPPH test, 55.96\%, is similar to that recorded previously for lime $(63.48 \%)$ and rape honey $(55.16 \%)$ [27].

Several studies indicate that there is a correlation between the total phenolic and flavonoids content and antiradical activity of honey - the higher content of phenolics results in higher antiradical activity [29,30]. Yellow sweet clover honey contains $67.55 \mathrm{mg}$ of GAE/100 $\mathrm{g}$ and $2.35 \mathrm{mg}$ of QE/100 g, while its antiradical activity is $55.96 \%$. This result correlates well with literature data.

\section{Materials and Methods}

\subsection{Reagents and Honey Samples}

All chemicals were of analytical grade. Methanol, dichloromethane, ethanol dibasic sodium phosphate heptahydrate $\left(\mathrm{Na}_{2} \mathrm{HPO}_{4} \times 7 \mathrm{H}_{2} \mathrm{O}\right)$, hydrochloric acid, and $85 \%$ phosphoric acid were purchased from POCH S.A. (Gliwice, Poland). Fifteen flavonoids: Apigenin, (+)-catechin, (-)-epicatechin, formononetin, galangin, genistein, kaempferol, chrysin, quercetin, morin hydrate, myricetin, (+)-naringenin, pelargonidin chloride, pinocembrin, and rutin hydrate; as well as 11 phenolic acids: 3-hydroxybenzoic acid, 3,4-dihydroxybenzoic acid, 4-hydroxybenzoic acid, caffeic acid, cinnamic acid, chlorogenic acid, ellagic acid, ferulic acid, gallic acid, $p$-coumaric acid, rosmarinic acid, syringic acid, and vanillic acid, used as standards, were purchased from Sigma-Aldrich (Poznań, Poland). Additionally, sesquiterpene, $( \pm$ )-abscisic acid, and coumarin (Sigma-Aldrich, Poznań, Poland) were used as standards. Stock solutions of each standard were prepared with HPLC-grade methanol at a concentration of $0.01 \mathrm{mmol} / \mathrm{L}$ and were stored at $4{ }^{\circ} \mathrm{C}$ with protection from light. Amberlite XAD-2, used as an adsorption resin for the extraction step, was obtained from Supelco (Bellefonte, PA, USA).

This study was carried out with nine yellow sweet clover honeys (Melilotus officinalis L.) obtained from professional beekeepers from the southeastern part of Poland (Subcarpathian Voivodeship) in three successive years: 2014, 2015, and 2016. The honey type was declared by the producers, based on the availability of nectar sources, location of the beehives, and sensory characteristics. All of the samples, after acquisition from beekeepers, were stored in hermetically sealed glass jars, in the dark, at $4{ }^{\circ} \mathrm{C}$.

\subsection{Extraction and Determination of Volatile Compounds}

\subsubsection{Steam distillation}

For steam distillation, $150 \mathrm{~g}$ of a Polish honey sample was dissolved in $150 \mathrm{~mL}$ distilled water and transferred into a $500 \mathrm{~mL}$ round-bottomed flask. The distillation time was $3 \mathrm{~h}$ in every case, and was repeated three times with the same amount of honey. The obtained distillate was transferred into a separation funnel and the aqueous phase was extracted with three portions of dichloromethane (total volume used for extraction was $400 \mathrm{~mL}$ ). Organic layers were combined and filtered through layers of anhydrous magnesium sulfate. All fractions were filtered through anhydrous magnesium sulfate and concentrated on a rotary evaporator at $35^{\circ} \mathrm{C}$ to a final volume of $100 \mu \mathrm{L}$, and $1 \mu \mathrm{L}$ was analyzed using GC-MS. The entire procedure was performed three times for each honey sample.

\subsubsection{Soxhlet Extraction}

Fifty-five grams of a honey sample was placed in a cellulose thimble and placed in a Soxhlet extraction apparatus containing $150 \mathrm{~mL}$ of dichloromethane and extraction was carried out for $10 \mathrm{~h}$. Obtained samples were filtered through anhydrous magnesium sulfate and concentrated on a rotary 
evaporator at $35^{\circ} \mathrm{C}$ to a final volume of $100 \mu \mathrm{L}$, and $1 \mu \mathrm{L}$ was analyzed using GC-MS. The procedure was performed three times for each sample.

\subsubsection{Ultrasound Solvent Extraction (USE)}

USE was performed as described previously [31] with some modifications. Forty grams of Polish sweet yellow clover honey sample were dissolved in $22 \mathrm{~mL}$ of distilled water in a 150-mL flask. Magnesium sulphate (1.5 g) was added and each sample was extensively vortexed. Then, $20 \mathrm{~mL}$ of dichloromethane was used as the extraction solvent for each honey sample. USE was performed in an ultrasound cleaning bath (Cole-Parmer 8891) at $25{ }^{\circ} \mathrm{C}$ for $30 \mathrm{~min}$. After the end of each sonication, the sample was introduced via a separation funnel and $20 \mathrm{~mL}$ of a saturated solution of $\mathrm{NaCl}$ was added for separation of organic and aqueous phases at room temperature. When the two layers were separated, the emulsion was collected. The whole extract was centrifuged at $3000 \mathrm{rpm}$, and the organic layer was collected and filtered through anhydrous magnesium sulphate. The aqueous layer was returned to the flask and another batch of $20 \mathrm{~mL}$ of dichloromethane was added. Each sample was extracted three times. Obtained fractions were filtered through anhydrous magnesium sulfate and concentrated on a rotary evaporator at $35^{\circ} \mathrm{C}$ to a final volume of $100 \mu \mathrm{L}$, and $1 \mu \mathrm{L}$ was analyzed using GC-MS. The entire procedure was carried out in triplicate for each honey sample.

\subsubsection{Solid Phase Extraction (SPE)}

SPE was done as described previously [32] with some modifications and was performed in a Baker SPE 12 g vacuum model (J. T. Baker ${ }^{\circledR}$, Phillipsburg, NJ, USA) with a flow rate of $2 \mathrm{~mL} / \mathrm{min}$. Strata-SDBL with $200 \mathrm{mg}$ of styrene-divinylbenzene resin cartridges (Phenomenex) were used for analysis. Prior to use, cartridges were conditioned by rinsing with $4 \mathrm{~mL}$ of dichloromethane, $4 \mathrm{~mL}$ of water, and $4 \mathrm{~mL}$ of ethanol-water mixture $(12 \%, v / v)$. Then, $15 \mathrm{~g}$ of honey sample dissolved in water was rinsed through the cartridges, and, subsequently, sugars and other hydrophilic components were washed with $20 \mathrm{~mL}$ of water; finally, elution was performed with $20 \mathrm{~mL}$ of dichloromethane. Obtained fractions were filtered through anhydrous magnesium sulfate and concentrated on a rotary evaporator at $35^{\circ} \mathrm{C}$ to a final volume of $100 \mu \mathrm{L}$, and $1 \mu \mathrm{L}$ was analyzed by GC-MS. The whole procedure was carried out in triplicate for each sample.

\subsubsection{Quantification of Coumarin Content by GC-MS}

Concentration of the coumarin in Polish sweet yellow clover honey samples was performed with respect to a standard curve. A standard stock solution of $1 \mathrm{mg} / \mathrm{mL}$ of coumarin was prepared in dichloromethane. The calibration curve was constructed for seven different concentrations of coumarin standards, three replications each. The equation used to calculate the amount of coumarin in sweet yellow clover honey samples was set as: $y=1,072,297 x-3,941,898\left(R^{2}=0.994\right)$. Repeatability of analyses was assessed using the same analysis under the same analytical conditions.

\subsubsection{GC-MS Analysis}

Analyses using gas chromatography-mass spectrometry were carried out with an HP 6890 GC System (Hewlett Packard, Böblingen, Germany) coupled to a 5973 Mass Selective Detector (Hewlett Packard, Böblingen, Germany). The mass detector worked in the electron impact ion-isolation mode at $70 \mathrm{eV}$, the mass range was $10-600$ units, and the ion source temperature was $230{ }^{\circ} \mathrm{C}$. A total of $1 \mu \mathrm{L}$ of extract was injected into the GC equipped with a $30 \mathrm{~m} \times 0.25 \mathrm{~mm}$ i.d. capillary column with an HP-5MS ((5\%-phenyl)-methylpolysiloxane, Agilent J and W GC column) with a coating thickness of $0.25 \mu \mathrm{m}$. Helium, at a constant flow rate of $2 \mathrm{~mL} / \mathrm{min}$, was used as the carrier gas, while chromatographic conditions were set as follows. Oven temperature was initially held at $40{ }^{\circ} \mathrm{C}$ for $3 \mathrm{~min}$, and then increased to $180{ }^{\circ} \mathrm{C}$ at a rate of $5^{\circ} \mathrm{C} / \mathrm{min}$, and, finally, increased to $250{ }^{\circ} \mathrm{C}$ at a rate of $10{ }^{\circ} \mathrm{C} / \mathrm{min}$. The equilibration time was $0.5 \mathrm{~min}$. The components were identified by comparison of their mass spectra with those of the spectrometer database using the NIST library (National Institute of Standards and 
Technology, Gaithersburg, MD, USA), with probabilities higher than $80 \%$. Each sample was analyzed three times.

\subsection{Extraction and Determination of Phenolic Compounds}

Phenolic compounds were extracted as described previously [33] with some modifications. Twenty-five grams of honey were dissolved in $125 \mathrm{~mL}$ of acidified water $(\mathrm{pH}=2$ with $\mathrm{HCl}$ ) and homogenized in an ultrasonic bath for $1 \mathrm{~h}$ until a nearly-clear fluid was obtained. The sample was then filtered through filter paper to remove any solid particles. Simultaneously, $40 \mathrm{~g}$ of Amberlite XAD-2 (Supelco, Bellefonte, PA, USA, pore size: $9 \mathrm{~nm}$, particle size: $0.3-1.2 \mathrm{~mm}$ ) was soaked in methanol and stirred with a magnetic stirrer for $30 \mathrm{~min}$. The mixture was allowed to stand for $10 \mathrm{~min}$ and was packed into a glass column $(2.5 \mathrm{~cm} \times 55 \mathrm{~cm})$. Next, the honey solution was transferred to a glass column and allowed to stand for 30-45 $\mathrm{min}$ in order to absorb honey phenolic compounds. The column was washed with acidified water $(0.05 \mathrm{M} \mathrm{HCl}, 250 \mathrm{~mL})$ and then distilled water $(300 \mathrm{~mL})$ to remove all sugars and other polar compounds. The flavonoids and phenolic compounds were eluted with methanol $(500 \mathrm{~mL})$ and the phenolic fraction was evaporated under reduced pressure at $40{ }^{\circ} \mathrm{C}$. The residue was then dissolved in $2 \mathrm{~mL}$ of methanol (HPLC-grade) and analyzed by HPLC with photodiode array (PDA) detection. The solution was evaporated again and the obtained residue was extracted three times with diethyl ether $(15 \mathrm{~mL})$. The remains were redissolved in $2 \mathrm{~mL}$ methanol (HPLC-grade) and analyzed by HPLC again.

\subsubsection{HPLC/PAD Analysis}

Analyses of the honey extracts were performed using an Ultimate 3000 Dionex HPLC system with a photodiode array detector (PDA) (Germering, Germany) and a Gemini $5 \mu \mathrm{m}$ C18 reverse phase column $(250 \times 4.6 \mathrm{~mm}$, particle size $5 \mu \mathrm{m})$. The mobile phase consisted of methanol (solvent A) and $0.01 \mathrm{M}$ aqueous phosphate buffer at $\mathrm{pH}=2.5$ (solvent $\mathrm{B}$ ) at a flow rate of $1 \mathrm{~mL} / \mathrm{min}$. The gradient separation was performed as follows (A:B ratio): $10 \%-90 \% t=0 ; 40 \%-60 \% t=13.5 \mathrm{~min}$; $90 \%-10 \% \mathrm{t}=39 \mathrm{~min} ; 100 \%-0 \% \mathrm{t}=42 \mathrm{~min} ; 10 \%-90 \% \mathrm{t}=55 \mathrm{~min}$. The analyses were carried out at $30{ }^{\circ} \mathrm{C}$. The chromatograms were recorded at 214 and $280 \mathrm{~nm}$, since most honey flavonoids and phenolic acids show their UV absorption maxima within the range of these wavelengths. The identification of phenolic compounds present in the honey extracts were based on the comparison of UV spectra and retention times with those found for standard compounds.

\subsubsection{Total Phenolic and Flavonoids Content}

The Folin-Ciocalteu method was used to spectrophotometrically determine the TPC [27]. Briefly, $5 \mathrm{~mL}$ of honey extracts $(0.5 \mathrm{~g}$ honey $/ 50 \mathrm{~mL}$ of distilled water) were mixed with $0.5 \mathrm{~mL}$ Folin-Ciocalteu reagent (FC) and $1.5 \mathrm{~mL} 20 \% \mathrm{Na}_{2} \mathrm{CO}_{3}$ solution and were placed into a 10 -mL volumetric flask. Then, they were diluted to $10 \mathrm{~mL}$ and kept in the dark for $120 \mathrm{~min}$. After this time, absorbance was measured at $760 \mathrm{~nm}$ (Spectrophotometer Hitachi U-2810, Tokyo, Japan) against the blank (water). TPC was expressed in terms of milligrams of gallic acid equivalents $\mathrm{mg}$ per $100 \mathrm{~g}$ honey and was determined as the average from three parallel measurements.

The TFC was determined using a colorimetric method [34]. Briefly, $1 \mathrm{~mL}$ of honey extract $(50 \mathrm{~g}$ honey $/ 50 \mathrm{~mL}$ of methanol) was mixed with $1 \mathrm{~mL} \mathrm{AlCl} 3$ solution $(10 \%)$. The mixture was allowed to stand for $60 \mathrm{~min}$. Absorbance was measured at $420 \mathrm{~nm}$ against the blank (mixture of methanol and reagent). Results were expressed as quercetin equivalents in milligrams per $100 \mathrm{~g}$ honey, and determined as the average from three parallel measurements.

\subsubsection{Antiradical Activity (DPPH Test)}

The antiradical activity extract of honey was evaluated using a modified method of Meda et al. [34]. Briefly, $1 \mathrm{~mL}$ of methanolic honey extract ( $10 \mathrm{~g}$ honey $/ 50 \mathrm{~mL}$ of methanol) was mixed with $4 \mathrm{~mL}$ of a methanolic solution of DPPH radicals $(0.1 \mathrm{mM})$. The control test was performed with a 
methanol-lacking extract. The mixture was mixed and allowed to stand in the dark for $30 \mathrm{~min}$. Absorbance was measured at $517 \mathrm{~nm}$ against the blank (methanol). Antiradical activity (\%) of the honey samples was calculated using the following formula:

$$
\mathrm{AA}[\%]=100 \times\left(\mathrm{Abs}_{\text {control }}-\mathrm{Abs}_{\text {sample }}\right) / \mathrm{Abs}_{\text {control }}
$$

where Abs represents the absorbance.

\section{Conclusions}

The HPLC-PAD data of the first investigation of the composition of unifloral M. officinalis honey revealed the dominant content of gallic acid and (+)-catechin. Their contents, and relative amounts, in the analyzed honey samples were constant and may be proposed as useful chemical markers of the floral origin of this honey. Furthermore, GC-MS analysis of the honey extracts showed the presence of interesting substances: Pharmacologically-active coumarin, which has, so far, been described as a main ingredient of French lavender honeys, and lumichrome, a derivative of the vitamin riboflavin, suggested as a marker among Dalmatian sage (S. officinalis L.) unifloral honey. The results of analysis of the tested honey samples clearly indicate that the combined presence of these compounds, alongside the high level of gallic acid and (+)-catechin (all of these compounds are shown in Figure 1), could be characteristic chemical fingerprints used to distinguish Polish yellow sweet clover honey from other unifloral honeys.

Acknowledgments: This research was supported by National Science Centre Project 2014/2015/ 15/B/NZ9/02182.

Author Contributions: I.J.-M. designed the experiments. I.J.-M., E.M. and N.S. performed the experiments. I.J.-M., E.M. and N.S. analyzed the data. All the authors discussed and planned the paper. I.J.-M. and E.M. drafted the manuscript.

Conflicts of Interest: The authors declare no conflict of interest.

\section{References}

1. Bunney, S. The Illustrated Encyclopedia of Herb. Their Medicinal and Culinary Uses; Chancellor Press: London, UK, 1992; pp. 190-198.

2. Chorepsima, S.; Tentolouris, K.; Dimitroulis, D.; Tentolouris, N. Melilotus: Contribution to wound healing in the diabetic foot. J. Herb. Med. 2013, 3, 81-86. [CrossRef]

3. Matławska, I. Leki roślinne w terapii guzków krwawniczych odbytu. Herbal remedies in the treatment of hemorrhoids. Postępy Fitoter. 2002, 3-4, 70-74.

4. Ehlers, D.; Platte, S.; Bork, W.R.; Gerard, D.; Quirin, K.W. HPLC analysis of sweet clover extracts. Deut. Lebensm. Rundsch. 1997, 93, 77-79.

5. Keating, G.; O'Kennedy, R. The chemistry and occurrence of coumarins. In Coumarins—Biology, Applications and Mode of Action; O'Kennedy, R., Thornes, R.D., Eds.; John Wiley \& Sons Ltd.: Chichester, UK, 1997; pp. 315-323.

6. Smith, W.K.; Gorz, H.J. Sweet clover improvement. Adv. Agron. 1965, 17, 163-231.

7. Locatelli, M.; Zengin, G.; Uysal, A.; Carradori, S.; de Luca, E.; Bellagamba, G.; Aktumsek, A.; Lazarova, I. Multicomponent pattern and biological activities of seven Asphodeline taxa: Potential sources of natural-functional ingredients for bioactive formulations. J. Enzyme Inhib. Med. Chem. 2016. [CrossRef] [PubMed]

8. Uysal, A.; Zengin, G.; Mollica, A.; Gunes, E.; Locatelli, M.; Yilmaz, T.; Aktumsek, A. Chemical and biological insights on Cotoneaster integerrimus: A new (-)-epicatechin source for food and medicinal applications. Phytomedicine 2016, 23, 979-988. [CrossRef] [PubMed]

9. Zengin, G.; Locatelli, M.; Ceylan, R.; Aktumsek, A. Anthraquinone profile, antioxidant and enzyme inhibitory effect of root extracts of eight Asphodeline taxa from Turkey: Can Asphodeline roots be considered as a new source of natural compounds? J. Enzyme Inhib. Med. Chem. 2016, 31, 754-759. [CrossRef] [PubMed] 
10. Lake, B. Coumarin metabolism, toxicity and carcinogenicity: Relevance for human risk assessment. Food Chem. Toxicol. 1999, 37, 423-453. [CrossRef]

11. Thada, R.; Chockalingam, S.; Dhandapani, R.K.; Panchamoorthy, R. Extraction and quantitation of coumarin from cinnamon and its effect on enzymatic browning in fresh apple juice: A bioinformatics approach to illuminate its antibrowning activity. J. Agric. Food Chem. 2013, 61, 5385-5390. [CrossRef] [PubMed]

12. Jerković, I.; Marijanović, Z.; Staver, M.M. Screening of natural organic volatiles from Prunus mahaleb L. Honey: Coumarin and vomifoliol as nonspecific biomarkers. Molecules 2011, 16, 2507-2518. [PubMed]

13. Guyot-Declerck, C. Floral quality and discrimination of Lavandula stoechas, Lavandula angustifolia, and Lavandula angustifolia x latifolia honeys. Food Chem. 2002, 79, 453-459. [CrossRef]

14. Martino, E.; Ramaiola, I.; Urbano, M.; Bracco, F.; Collina, S. Microwave-assisted extraction of coumarin and related compounds from Melilotus officinalis (L.) Pallas as an alternative to Soxhlet and ultrasound-assisted extraction. J. Chromatogr. A 2006, 1125, 147-151. [CrossRef] [PubMed]

15. Bouseta, A.; Scheirman, V.; Collin, S. Flavor and free amino acid composition of lavender and Eucalyptus honeys. J. Food Sci. 1996, 61, 683-687. [CrossRef]

16. Matiru, V.N.; Dakora, F.D. Xylem transport and shoot accumulation of lumichrome, a newly recognized rhizobial signal, alters root respiration, stomatal conductance, leaf transpiration and photosynthetic rates in legumes and cereals. New Phytol. 2005, 165, 847-855. [CrossRef] [PubMed]

17. Tuberoso, C.I.G.; Jerković, I.; Bifulco, E.; Marijanovic, Z.; Congiu, F.; Bubalo, D. Riboflavin and lumichrome in Dalmatian sage honey and other unifloral honeys determined by LC-DAD technique. Food Chem. 2012, 135, 1985-1990. [CrossRef] [PubMed]

18. Tuberoso, C.I.G.; Bifulco, E.; Caboni, P.; Sarais, G.; Cottiglia, F.; Floris, I. Lumichrome and phenyllactic acid as chemical markers of thistle (Galactites tomentosa Moench) honey. J. Agric. Food Chem. 2011, 59, 364-369. [CrossRef] [PubMed]

19. Kuś, P.M.; Jerković, I.; Tuberoso, C.I.G.; Marijanović, Z.; Congiu, F. Cornflower (Centaurea cyanus L.) honey quality parameters: Chromatographic fingerprints, chemical biomarkers, antioxidant capacity and others. Food Chem. 2014, 142, 12-18.

20. Jasicka-Misiak, I.; Poliwoda, A.; Dereń, M.; Kafarski, P. Phenolic compounds and abscisic acid as potential markers for the floral origin of two Polish unifloral honeys. Food Chem. 2012, 131, 1149-1156. [CrossRef]

21. Can, Z.; Yildiz, O.; Sahin, H.; Turumtay, E.A.; Silici, S.; Kolayli, S. An investigation of Turkish honeys: Their physico-chemical properties, antioxidant capacities and phenolic profile. Food Chem. 2015, 180, 133-141. [CrossRef] [PubMed]

22. Piljac-Zegarac, J.; Stipcevic, T.; Belscak, A. Antioxidant properties and phenolic content of different floral origin honeys. J. Apiprod. Apimed. Sci. 2009, 1, 43-50. [CrossRef]

23. Dimitrova, B.; Gevrenova, R.; Anklam, E. Analysis of phenolic acids in honeys of different floral origin by solid-phase extraction and highperformance liquid chromatography. Phytochem. Anal. 2007, 18, 24-32. [CrossRef] [PubMed]

24. Khalil, M.I.; Alam, N.; Moniruzzaman, M.; Sulaiman, S.A.; Gan, S.H. Phenolic acid composition and antioxidant properties of Malaysian honeys. J. Food Sci. 2011, 76, 921-928. [CrossRef] [PubMed]

25. Hussein, S.Z.; Yusoff, K.M.; Makpol, S.; Yusof, Y.A.M. Antioxidant capacities and total phenolic contents increase with gamma irradiation in two types of Malaysian honey. Molecules 2011, 16, 6378-6395. [CrossRef] [PubMed]

26. Beretta, G.; Granata, P.; Ferrero, M.; Orioli, M.; Facino, R.M. Standardization of antioxidant properties of honey by a combination of spectrophotometric/fluorimetric assays and chemometrics. Anal. Chim. Acta 2005, 533, 185-191. [CrossRef]

27. Wilczyńska, A. Phenolic content and antioxidant activity of different types of Polish honey-A short report. Pol. J. Food Nutr. Sci. 2010, 60, 309-313.

28. Rababah, T.M.; Al-Omoush, M.; Brewer, S.; Alhamad, M.; Yang, W.; Alrababah, M.; Al-Ghzawi, A.A.; Al-u'datt, M.; Ereifej, K.; Alsheyab, F.; et al. Total phenol, antioxidant activity, flavonoids, anthocyanins and color of honey as affected by floral origin found in the arid and semiarid Mediterranean areas. J. Food Process. Preserv. 2014, 38, 1119-1128. [CrossRef] 
29. Da Silva, I.A.; da Silva, T.M.; Camara, C.A.; Queiroz, N.; Magnani, M.; de Novais, J.S.; Soledade, L.E.; Lima Ede, O.; de Souza, A.L.; de Souza, A.G. Phenolic profile, antioxidant activity and palynological analysis of stingless bee honey from Amazonas, Northern Brazil. Food Chem. 2013, 141, 3552-3558. [CrossRef] [PubMed]

30. Vulić, J.; Čanadanović-Brunet, J.; Ćetković, G.; Djilas, S.; Tumbas Šaponjac, V. Antioxidant and sensorial properties of polyfloral honey with dried apricots after one year of storage. J. Chem. 2015, 2015, 858049. [CrossRef]

31. Alissandrakis, E.; Daferera, D.; Tarantilis, P.A.; Polissiou, M.; Harizanis, P.C. Ultrasound-Assisted extraction of volatile compounds from citrus flowers and citrus honey. Food Chem. 2003, 82, 575-582. [CrossRef]

32. Castro-Vázquez, L.; Consuelo Díaz-Maroto, M.; Guchu, E.; Soledad Pérez-Coello, M. Analysis of volatile compounds of eucalyptus honey by solid phase extraction followed by gas chromatography coupled to mass spectrometry. Eur. Food Res. Technol. 2006, 224, 27-31. [CrossRef]

33. Martos, I.; Cossentini, M.; Ferreres, F.; Tomas-Barberan, F.A. Flavonoid composition of Tunisian honeys and propolis. J. Agric. Food Chem. 1997, 45, 2824-2829. [CrossRef]

34. Meda, A.; Lamien, Ch.; Romito, M.; Millogo, J.; Nacoulma, O. Determination of the total phenolic, flavonoid and proline contents in Burkina Fasan honey, as well as their radical scavenging activity. Food Chem. 2005, 91, 571-577. [CrossRef]

Sample Availability: The honey samples are available from the authors for limited time.

(C) 2017 by the authors; licensee MDPI, Basel, Switzerland. This article is an open access article distributed under the terms and conditions of the Creative Commons Attribution (CC-BY) license (http:/ / creativecommons.org/licenses/by/4.0/). 